\title{
Filter implementation technique for multicriteria characterization of coding domains in the joint transform correlator
}

\author{
Laurent Bigué and Pierre Ambs
}

\begin{abstract}
An improved method for implementing correlation filters in the joint transform correlator architecture is proposed. We derived the method from computer-generated holography techniques. It allows us to use any correlation filters, especially ones that provide an optimal trade-off between noise robustness, peak sharpness, and optical efficiency, with any spatial light modulator (SLM). This method also allows for an objective comparison of the performance of the coding domains of various SLM's. (C) 1999 Optical Society of America
\end{abstract}

OCIS codes: $\quad 070.5010,070.4550,230.6120$.

\section{Introduction}

Since the inception of optical correlation under coherent illumination, ${ }^{1-3}$ many correlation filters have been proposed ${ }^{4-6}$ (see Ref. 7 for a review). Yet some problems remain: These filters are to be implemented into optical architectures, and they are displayed by means of spatial light modulators (SLM's). These SLM's can encode only a limited number of values called the coding domain, and in most cases the filter values do not fit the coding domain. The question of implementing correlation filters onto SLM's in the $4 f$ architecture has already been considered, ${ }^{8-10}$ and optimal solutions have been proposed. In the joint transform correlator (JTC) architecture the question is still open, because in the case of highly restricted coding domains (e.g., binary or ternary) the adaptation of the previous technique seldom succeeds. Nevertheless, such adaptation proves of interest: It gives a general framework that allows a user an objective comparison of different filters and of various coding domains.

Laude and Réfrégier ${ }^{10}$ also answered a very important question: Which coding domain (which SLM)

The authors are with the Université de Haute Alsace, École Supérieure des Sciences Appliquées pour l'Ingénieur-Mulhouse, 12, rue des Frères Lumière, 68093 Mulhouse, Cedex France. L. Bigué's e-mail address is L.Bigue@essaim.univ-mulhouse.fr.

Received 27 March 1998; revised manuscript received 15 March 1999.

0003-6935/99/204296-10\$15.00/0

(C) 1999 Optical Society of America performs best? Such a question is important when it comes to choosing a SLM for a particular application. This important question is still unanswered in the case of the JTC.

The filter performance can be evaluated through the estimation of several metrics: The sharpness of the correlation peak, noise robustness, and optical efficiency are usually considered; Juday ${ }^{9}$ proposed another criterion for evaluating peak sharpness, and Vijaya Kumar et al.11 also suggested the response uniformity in the case of a composite distortiontolerant filter (this list is not exhaustive). Usually comparing coding domains amounts to comparing particular filters with different regions of support. The results provided by this technique rely too much on the type of filter considered and should be avoided. A better idea, rather, is to synthesize optimal tradeoff (OT) filters (considering $N$ criteria; these filters, given $N-1$ criteria, will optimize the last criterion). Synthesizing a complete set of OT filters gives a range of filters with quite different properties. The implementations of all the filters into the set on the various coding domains can be compared and will give a comparison of the various coding domains over a large number of different filters.

Our purpose in this paper is to propose a technique that allows for as good a filter implementation as possible into the JTC (the implementation of the scene is not within the scope of this paper, but it can be considered with much interest as discussed below). In Section 2 we review the state of the art concerning OT filters. In Section 3 we describe the method we suggest for the implementation onto limited coding 
domains. Section 4 gives examples of such implementations. In Section 5 we apply this technique to the comparison of coding domains provided by various SLM's. Finally, Section 6 consists of a discussion about coding the scene.

\section{Optimal Trade-Off Filters: Review}

In the following-for clarity-images will be represented as one-dimensional vectors obtained by a lexicographical reading.

Let us mention the basic principle of OT filters. ${ }^{6}$ We consider correlation filters $\mathbf{h}$ (or $\hat{\mathbf{h}}$ in the Fourier space) that correspond to a reference image r. For historical reasons one of the most popular filters is the matched filter expressed as

$$
\left(\hat{\mathbf{h}}_{M}\right)_{k}=\frac{\hat{\mathbf{r}}_{k}^{*}}{\hat{S}_{k}},
$$

where $\hat{S}$ denotes the power spectral density of the noise the filter is matched to and $*$ is the complex conjugate. It is shown that this filter minimizes the mean-squared error expressed as

$$
\mathrm{MSE}=\sum_{k}{\hat{S_{k}}}_{k}\left|\hat{\mathbf{h}}_{k}\right|^{2} .
$$

Many other filters have been proposed. We notice among them the inverse filter that optimizes peak sharpness. We will characterize peak sharpness through the computation of the correlation plane energy (CPE) that must be all the lower, since the peak is sharp. CPE can be expressed as

$$
\mathrm{CPE}=\sum_{k} \hat{D}_{k}\left|\hat{\mathbf{h}}_{k}\right|^{2},
$$

where $\hat{D}$ is the power spectral density of the reference image $\left(\hat{D}_{k}=\left|\hat{\mathbf{r}}_{k}\right|^{2}\right) \mathbf{r}$. Then the inverse filter is expressed as follows:

$$
\left(\hat{\mathbf{h}}_{I}\right)_{k}=\frac{\hat{\mathbf{r}}_{k}^{*}}{\hat{D}_{k}}=\frac{\hat{\mathbf{r}}_{k}^{*}}{\left|\hat{\mathbf{r}}_{k}\right|^{2}} .
$$

Another important criterion is the filter optical efficiency $\eta$ :

$$
\eta=\left|\hat{\mathbf{h}}^{+} \cdot \hat{\mathbf{r}}\right|^{2}=\left|\sum_{k} \hat{\mathbf{h}}_{k}^{*} \hat{\mathbf{r}}_{k}\right|^{2},
$$

where ${ }^{+}$denotes the complex-conjugate transposition operator and the raised dot denotes the scalar product between two vectors.

This criterion is optimized by the phase-only filter $^{12}$ :

$$
\left(\hat{\mathbf{h}}_{\mathrm{PO}}\right)_{k}=\frac{\hat{\mathbf{r}}_{k}^{*}}{\left|\hat{\mathbf{r}}_{k}\right|} .
$$

The filters presented above prove quite efficient as far as the criterion they optimized is concerned. Conversely, they exhibit little performance for other criteria. ${ }^{13}$ In such cases it can be interesting to synthesize a filter whose behavior is intermediate. A simple linear combination of the three abovementioned filters could be convenient, but Réfrégier ${ }^{14}$ proposes instead a filter called the OT filter that, given two criteria, optimizes the latter one. It can be shown that this filter minimizes the linear combination of criteria $E(\hat{\mathbf{h}})$ :

$$
E(\hat{\mathbf{h}})=\alpha \mathrm{MSE}+\beta \mathrm{CPE}-2 \gamma \eta,
$$

where $(\alpha, \beta, 2 \gamma)$ are the coordinates of a unity vector $\mathbf{u}$ of the first quadrant. This filter is expressed as

$$
\left(\hat{\mathbf{h}}_{\mathrm{OT}}\right)_{k}=\gamma \frac{\hat{\mathbf{r}}_{k}^{*}}{\hat{B}_{k}},
$$

where

$$
\hat{B}_{\mathbf{u}}=\alpha \hat{S}+\beta \hat{D} .
$$

In some cases the use of modified criteria, such as signal-to-noise ratio (SNR) or peak-to-background correlation energy (PBCE), is preferable. They can be expressed as

$$
\begin{gathered}
\operatorname{SNR}(\mathbf{h})=\frac{\eta(\mathbf{h})}{\operatorname{MSE}(\mathbf{h})}=\frac{\left|\sum_{k} \hat{\mathbf{h}}_{k}^{*} \hat{\mathbf{r}}_{k}\right|^{2}}{\sum_{k} \hat{S}_{k}\left|\hat{\mathbf{h}}_{k}\right|^{2}}, \\
\operatorname{PBCE}(\mathbf{h})=\frac{\eta(\mathbf{h})}{\operatorname{CPE}(\mathbf{h})}=\frac{\left|\sum_{k} \hat{\mathbf{h}}_{k}^{*} \hat{\mathbf{r}}_{k}\right|^{2}}{\sum_{k} \hat{D}_{k}\left|\hat{\mathbf{h}}_{k}\right|^{2}} .
\end{gathered}
$$

Drawing the locus of points (SNR, PBCE, $\eta$ ) when $\mathbf{u}$ describes the first quadrant gives a hypersurface of the criteria space. In the case in which three criteria are considered, the locus is a three-dimensional surface called the optimal characteristics surface (OCS). Since for $N-1$ given criteria it maximizes the $N$ th criterion, this surface is convex. In the present case, in which no constraint is applied to the filter (any point of the filter can take any complex value), there is no point in tracing the whole OCS, since SNR and PBCE are independent of $\eta$. The only interesting figures are then the two-dimensional (2D) cross section (SNR, PBCE) of the OCS.

\section{Implementation of Optimal Trade-Off Filters onto Limited Coding Domains}

The implementation of correlation filters is performed with SLM's, characterized by a coding domain (i.e., the complex values it can produce). Some examples of coding domains are given Fig. 1, and a review of existing modulation techniques can be found in Ref. 15.

Actually, implementing correlation filters onto modulators with limited capabilities is an old problem that has already led to a large number of publications. Two methods can be considered: Either the filter can be directly calculated in its final form (binary or ternary, for example), ${ }^{16-18}$ or an ideal continuous-valued filter is first computed and then 


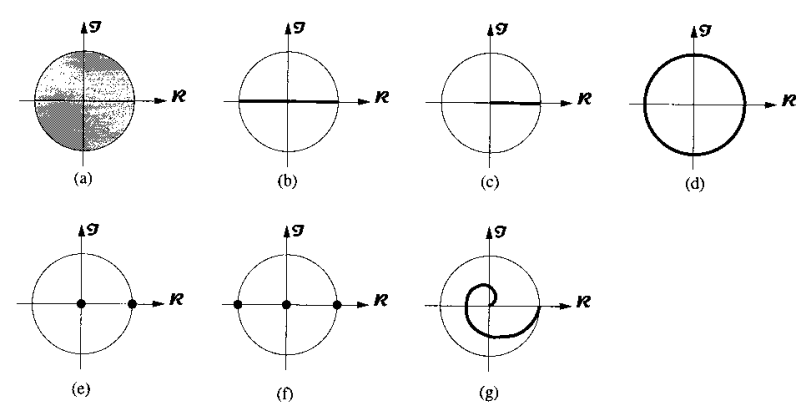

Fig. 1. Examples of coding domains: (a) unity disk, (b) real axis $([-1 ; 1])$, (c) amplitude only $([0 ; 1]),(\mathrm{d})$ phase only, (e) binary, (f) ternary, (g) spiral.

constrained onto the coding domain of the modulator. ${ }^{9,10}$ We will use the latter, which is probably more complicated but nevertheless more versatile: Some direct methods are often dedicated to given coding domains and cannot be extended to other domains in a simple way. Other considerations then show that our chosen method produces filters that are at least as good as those produced with the first technique (by construction, an OT filter that is optimally implemented is the best possible implementation according to a given number of criteria).

As stated above, correlation filters and especially OT filters such as those mentioned above, present a large range of values, real or complex, that most SLM's cannot reproduce. In that case the best solution ${ }^{10}$ is to make the filter fit the SLM coding domain: The filter must be processed to have only a restricted number of different values.

Let us consider the same three criteria as in Section 2. We denote the unconstrained OT filter related to the compromise vector $\mathbf{u}(\alpha, \beta, 2 \gamma) \hat{\mathbf{h}}_{\mathbf{u}}{ }^{0}$, and its optimal implementation onto the coding domain $\hat{\mathbf{h}}$. It can be shown ${ }^{10}$ that $\hat{\mathbf{h}}$ minimizes the modified energy function:

$$
E_{\varphi}(\hat{\mathbf{h}})=\sum_{k}\left(\hat{B}_{\mathbf{u}}\right)_{k}\left|\hat{\mathbf{h}}_{k}-\left(\hat{\mathbf{h}}_{\mathbf{u}}{ }^{0}\right)_{k} \exp (i \varphi)\right|^{2}
$$

where $\varphi \in[0 ; 2 \pi]$ is an angular parameter to be optimized. This solution can be seen as a simplified version of the minimum Euclidean distance principle proposed by Juday, ${ }^{9}$ except that in this particular case the only parameter to be optimized is the dephasing angle $\varphi$ (the optimization of the gain parameter suggested by Juday is discussed in Section 4).

\section{A. Implementation into the $4 f$ Correlator}

The implementation of OT filters into such a correlator has already been considered. ${ }^{10}$ In the case in which there are three criteria (SNR, PBCE, and $\eta$ ) it is shown that the OT filter's $\hat{\mathbf{h}}_{\mathbf{u}}{ }^{0}$ optimal implementation $\hat{\mathbf{h}}$ minimizes the energy function:

$$
E_{\varphi}(\hat{\mathbf{h}})=\sum_{k}\left|\hat{\mathbf{h}}_{k}-\left(\hat{\mathbf{h}}_{\mathbf{u}}{ }^{0}\right)_{k} \exp (i \varphi)\right|^{2} .
$$

Though this optimization is performed through an iterative search, the required amount of computation remains low and $\hat{\mathbf{h}}$ can be easily computed: The implementation $\hat{\mathbf{h}}$ is no more than the Euclidean projection of $\hat{\mathbf{h}}_{\mathbf{u}}{ }^{0}$ (dephased by $\varphi$ ) onto the considered coding domain. Recently, Vijaya Kumar et al. ${ }^{19}$ proposed a simple method to compute this optimal angle.

\section{B. Implementation into the Joint Transform Correlator}

In the case of an implementation into the JTC the problem proves more complicated, because we display $\mathbf{h}$ and not $\hat{\mathbf{h}}$. The energy function $E(\hat{\mathbf{h}})$ must then be expressed in the direct domain. Through Parseval's theorem we obtain the following from Eq. (12):

$$
\begin{aligned}
E_{\varphi}(\mathbf{h})= & B_{\mathbf{u}}^{+} \cdot\left\{\left[\mathbf{h}-\mathbf{h}_{\mathbf{u}}{ }^{0} \exp (-i \varphi)\right]^{*}\right. \\
& \left.*\left[\mathbf{h}-\mathbf{h}_{\mathbf{u}}{ }^{0} \exp (-i \varphi)\right]\right\} \\
= & \sum_{k} \sum_{k^{\prime}}\left(B_{\mathbf{u}}\right)_{k} \cdot\left[\mathbf{h}_{k}-\left(\mathbf{h}_{\mathbf{u}}{ }^{0}\right)_{k} \exp (-i \varphi)\right] \\
& \cdot\left[\mathbf{h}_{k-k^{\prime}}-\left(\mathbf{h}_{\mathbf{u}}{ }^{0}\right)_{k-k^{\prime}} \exp (-i \varphi)\right]^{*},
\end{aligned}
$$

where $*$ denotes the convolution operator. No analytical solution clearly appears, except when $B_{\mathbf{u}}$ is a Dirac pulse, that is, when $\hat{B}_{\mathbf{u}}$ is uniform (similar to a white-noise power spectral density). In this trivial case Eq. (14) can be simplified as

$$
\begin{aligned}
E_{\varphi}(\mathbf{h}) & =\left[\mathbf{h}-\mathbf{h}_{\mathbf{u}}{ }^{0} \exp (-i \varphi)\right]^{*} \cdot\left[\mathbf{h}-\mathbf{h}_{\mathbf{u}}{ }^{0} \exp (-i \varphi)\right] \\
& =\sum_{k}\left|\hat{\mathbf{h}}_{k}-\left(\hat{\mathbf{h}}_{\mathbf{u}}{ }^{0}\right)_{k} \exp (i \varphi)\right|^{2} \\
& =\sum_{k}\left|\mathbf{h}_{k}-\left(\mathbf{h}_{\mathbf{u}}{ }^{0}\right)_{k} \exp (i \varphi)\right|^{2}
\end{aligned}
$$

and the solution similar to that used in the $4 f$ correlator is valid.

Nevertheless, in the most general case, the two summations are time consuming and will not allow for the iterative search of $\mathbf{h}$ from this expression. But we notice that our purpose is similar to that of computer-generated holography $(\mathrm{CGH})$ : to produce a given distribution in the direct domain with a distribution in the Fourier space that has a limited number of possible values. We will therefore manage to apply CGH techniques to our case.

\section{Computer-Generated Holography Techniques}

Since the inception of CGH in the late 1960's, numerous methods have been proposed. In general, the more accurate they are, the slower they are. We will detail only two of them, known to be the most efficient.

Generalized projection onto convex sets. This kind of method, not originally dedicated to holography, consists of performing successive direct and inverse Fourier transforms, each time preceded by constraint imposition: In the space domain a quantization is performed, and in the Fourier domain a windowing or an error reduction is achieved. From a historic viewpoint, Burch's technique ${ }^{20}$ and the iterative Fourier transform algorithm (IFTA) ${ }^{21}$ are the first ex- 
plicit implementations from the projection onto convex sets (POCS) family in the CGH domain. The IFTA was derived from the Gerchberg-Saxton algorithm, ${ }^{22}$ originally designed for the recovery of phase figures when only intensity figures are known in both direct and Fourier planes. In fact, all these algorithms are part of generalized POCS, ${ }^{23}$ whose first explicit theoretical formulation for $\mathrm{CGH}$ is found in Ref. 24. The POCS framework ${ }^{25}$ provides an efficient tool for $\mathrm{CGH}$ methods design, thanks to the variety of constraints it can take into account, including the progressive quantization in the hologram plane $^{21}$ and the progressive quantization of pixels chosen randomly. ${ }^{26}$

Direct binary search. The direct binary search (DBS ${ }^{27}$ amounts to performing a Monte Carlo technique. A random binary hologram is generated, and the error between the reconstruction produced by the hologram and the desired distribution is computed. Then, one after the other, each pixel is inverted, and for each inversion the error is computed again. The inversion is maintained if the error has decreased. This cycle is stopped when the error stops decreasing. This method is known to be quite powerful but also time consuming. It can be improved with the addition of simulated annealing, but the computation time becomes excessive. This method is attractive in the case in which the coding domains are limited in extent (e.g., binary) but should be avoided when the number of possible levels increases.

Hybrid technique. The comparison between DBS and IFTA/POCS is difficult, because there are many variants of each. Nevertheless, as reported by several authors, DBS generally proves more efficient than POCS, but it consumes far more computer time. ${ }^{28-30}$ The advantages of the two methods can be achieved when the two are combined. ${ }^{30}$ A few IFTA/POCS-like iterations are first performed, providing a preliminary hologram.

The hologram produced by IFTA/POCS is then used in DBS iterations instead of the initial random distribution. This implementation proves efficient (almost as efficient as DBS), although it remains far less computer intensive than the classic DBS technique (the required number of DBS iterations is reduced). Of course, such a combination is to be considered only in the case in which DBS can be applied and is not used in the case of continuous or many-leveled domains.

\section{Applications of Computer-Generated Hologram Techniques to Correlation Filter Implementation}

The previously described techniques require a few modifications before they can be used for correlation filter implementation. In that case the desired filter, expressed in the Fourier plane, corresponds to the reconstructed image in the $\mathrm{CGH}$ case and the filter implementation (in the direct domain) to the hologram. The error is replaced with the modified energy function of Eq. (12). Applying CGH techniques to correlation filter implementation has already been considered elsewhere, but the CGH

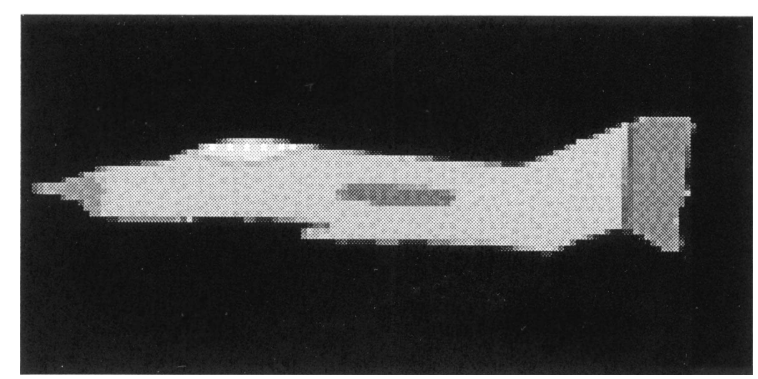

Fig. 2. Reference image used for synthesizing OT filters.

techniques used, often noniterative detour-phase methods ${ }^{31,32}$ similar to Lee's technique, ${ }^{33}$ show poorer results than do the methods presented above that take account of the minimum Euclidean distance principle. ${ }^{9}$ Below, a hybrid technique that combines IFTA/POCS and DBS is used.

Our POCS-like technique consists of a gradient projection algorithm, in which the classic projection is replaced with a progressive projection, as suggested by Wyrowski.21 Thus we call this algorithm gradient-progressive projection (GPP). It is extensively described in Appendixes A and B. Because of the addition of DBS iterations to GPP iterations when the extent of the coding domain remains limited (i.e., when the coding levels are discrete), in the following such a technique may be denoted GPP + DBS (this version of the algorithm is described in Appendix C).

\section{Examples of Implementations}

We computed a set of filters, which describe a large range of trade-offs $(\alpha, \beta, 2 \gamma)$, from the reference image depicted Fig. 2 and drew their OCS. Here drawing such a surface does not prove useful; $2 \mathrm{D}$ cross sections of these OCS's are more interesting. Each of them is a monotonic curve that is all the more optimal, since it will be close to the top right-hand corner of the figure (the point whose coordinates are the respective maxima of the two considered criteria). The criteria have been normalized to their maximum values, obtained with either the matched filter (SNR), the inverse filter (PBCE), or the phase filter $(\eta)$. As explained above, when no constraint is applied to the filters, the only interesting curve is the 2D cross section (SNR, PBCE) (Fig. 3).

For our simulations we chose a white-noise model (whereas our algorithm is not dedicated to a particular noise model). The two reasons for this choice are (i) literature about the $4 f$ correlator presents results that consider white noise (how to compare $4 f$ / JTC if we consider two different noise models) and (ii) choosing a white-noise model minimizes noise mismatching (mismatching occurs when the noise model that was taken into account during the filter synthesis does not correspond to the model of the noise that affects the scene).

We compared the method we advocate with the traditional noniterative projection in the case of a binary coding domain that constitutes the most strin- 


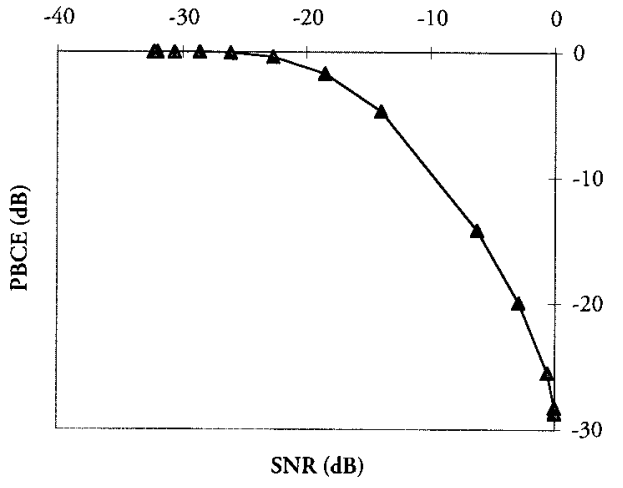

Fig. 3. Locus of points (SNR, PBCE). The filters are not constrained to a particular coding domain.

gent case. Figure 4 depicts the compromise between peak sharpness and efficiency. Clearly, the classic Euclidean projection behaves worse than our method GPP + DBS that provides a better compromise. In that particular case of a binary domain, GPP used alone does not work correctly [the locus (PBCE, $\eta$ ) is reduced to a single point] and does not allow a user to tune the compromise between peak sharpness and optical efficiency: Whatever the filter considered in the beginning, it converges to the same binary distribution.

As far as the compromise between noise resistance and efficiency is concerned (Fig. 5), we can notice that, in the best case, the classic projection shows the same performance as GPP + DBS. This is not surprising, since in this case (we chose a white-noise model) Eq. (14) reduces to Eq. (15) and a classical Euclidean projection is the simplest and the best solution. Once again, GPP used alone does not allow a user to tune the compromise between the considered criteria.

The most interesting curve may be the one that describes the trade-off between noise resistance and peak sharpness (Fig. 6). As expected, the classic Euclidean projection does not provide a genuine portion of OCS, since the curve is not monotonic. The compromise provided by GPP is better, because the curve looks like a real OCS part, and it is closer to the ideal

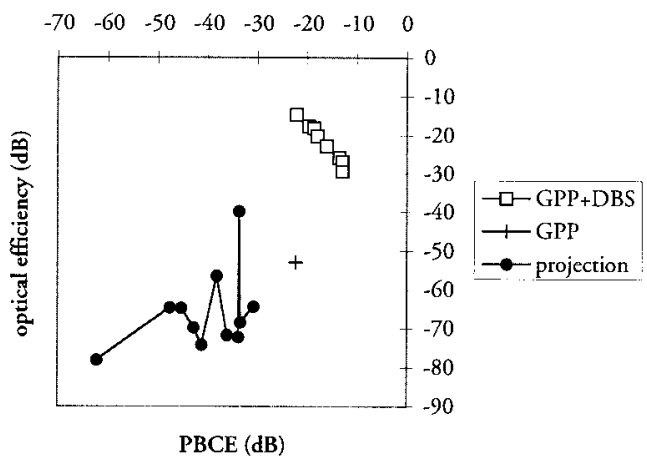

Fig. 4. Locus of points (PBCE, $\eta$ ) for $\alpha=0$ in the case in which the filter is constrained onto a binary domain. Various methods were investigated.

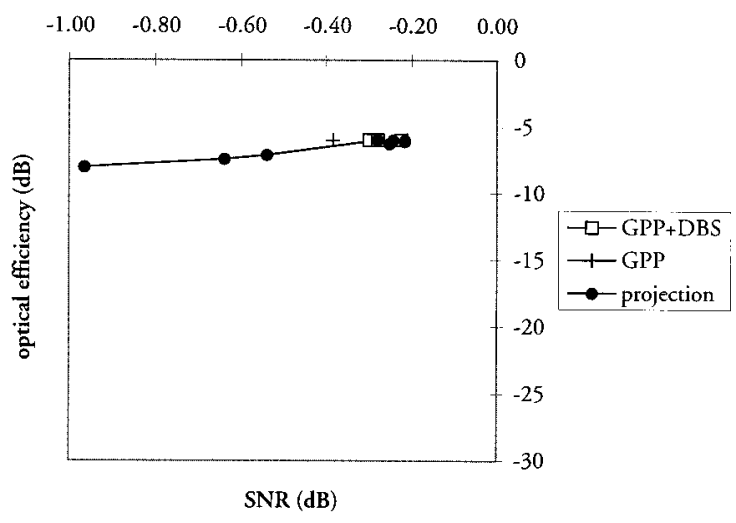

Fig. 5. Locus of points (SNR, $\eta$ ) for $\beta=0$ in the case in which the filter is constrained onto a binary domain. Various methods were investigated.

case. But as in the previous cases, using GPP + DBS proves to be the best solution. In the following, its use, when possible, is preferred.

One may object that sometimes GPP + DBS proves complicated and does not provide better performance than do simpler methods. Our response is that this technique can be used whatever the considered tuning of the compromise between the various criteria and that no other technique can perform better.

Let us compare our technique with Juday's suggestions ${ }^{9}$ : It finally amounts to performing an iterative search over the angular parameter $\varphi$ (when needed, in the case of the spiral coding domain). As far as the gain parameter $\gamma$ is concerned, it is either fixed directly (when we study the trade-off between PBCE and $\eta$ or between SNR and $\eta$ ), because in this case the optimization concerns a criterion other than $\eta$, or it is iteratively optimized when we study the trade-off between SNR and PBCE.

\section{Comparison of Coding Domains}

We implemented the previous set of filters onto limited coding domains and drew the locus of points (SNR, PBCE, $\eta$ ). Here the rigorous denomination OCS should not be used, because the surfaces do not

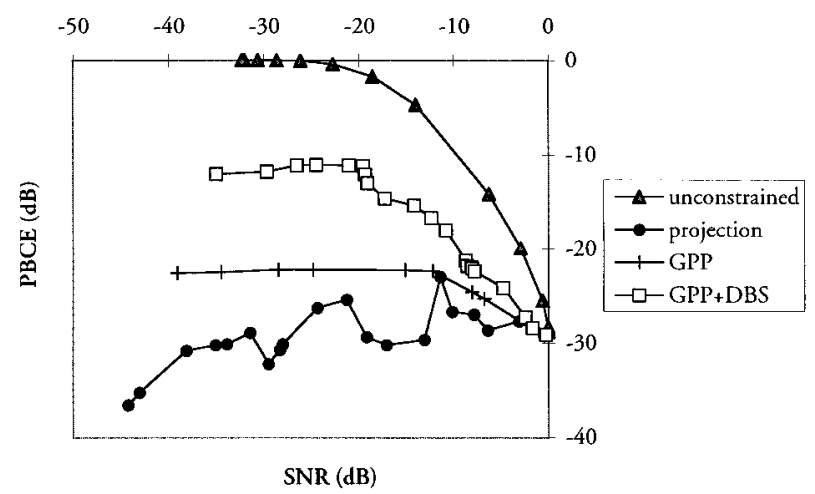

Fig. 6. Locus of points (SNR, PBCE) in the case in which the filter is constrained onto a binary domain ( $\gamma$ has been optimized). Various methods were investigated. 


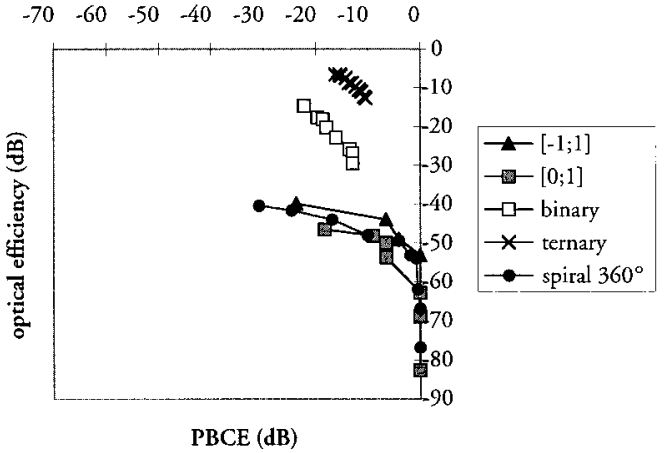

Fig. 7. Implementation of OT filters onto various coding domains. The curves describe the compromise between peak sharpness and optical efficiency.

a priori show the same monotony as does genuine OCS. Once again, 2D cross sections will be studied.

Figure 7 depicts the compromise between peak sharpness and efficiency. This trade-off is disappointing: In this particular case $(\alpha=0)$, it amounts to implementing inverse filters that are known to be unstable and hard to implement in $4 f$ architecture (regularization, i.e., limitation of the filter dynamic range, is often required). In the JTC architecture their implementation is also difficult. Our technique allows for the tuning of the trade-off, but optical efficiency remains low for continuous domains. We notice paradoxically that the binary and the ternary domains perform better than do continuous domains, but they are more extended: This can be explained simply, because of the numerical technique used for the implementation, which finely optimizes the convergence in the case in which the coding domain is restricted.

Figure 8 reports the study of the trade-off between noise resistance and optical efficiency $(\beta=0)$. The loci of the trade-off indicate that there is not much room to maneuver: A mere ternary coding allows a user to reach the most interesting part of the curve and to almost equal the performance of the continuous coding. This is not surprising, since the filters we implemented $(\beta=0)$ are matched filters and in

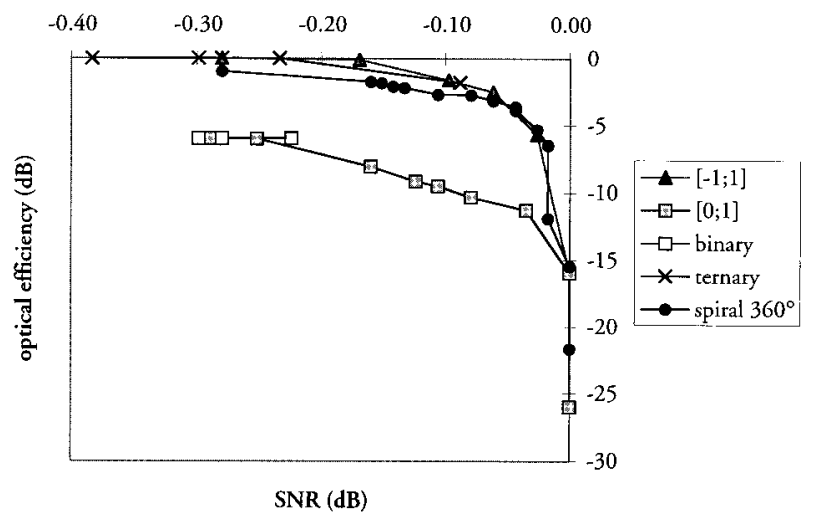

Fig. 8. Implementation of OT filters onto various coding domains. The curves describe the compromise between noise resistance and optical efficiency.

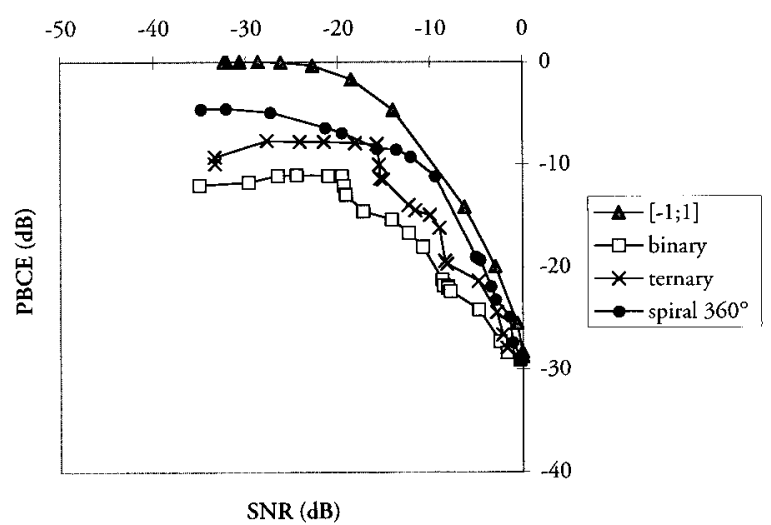

Fig. 9. Implementation of OT filters onto various coding domains. The curves describe the compromise between noise resistance and peak sharpness. As explained in Section 5, the results for $[0 ; 1]$ are not explicitly given.

our particular case are matched to white noise, as discussed in Section 4. Their aspect is therefore similar to that of the reference, and their gray-level histogram, instead of being quite continuous as in the general case of OT filters, involves a reduced number of rays (in this case five, two of which are much higher than the others). Then implementing these filters onto a limited coding domain (even with three levels) proves to be close to optimum.

Figure 9 reports the most interesting study, which concerns the trade-off between noise resistance and peak sharpness. In this case, a value is chosen for $\alpha$, and the value of $\beta$ is the one that optimizes the compromise. This involves optimizing the gain parameter as suggested by Vijaya Kumar et al..$^{8}$ it is also an iterative search. Results for domain $[0 ; 1]$ are not explicitly given, since it is equivalent to using domain $[0 ; 1]$ or $[-1 ; 1]$, except that the efficiency is not the same. The filter implemented onto $[0 ; 1]$ is simply derived from the one implemented onto $[-1 ; 1]$ by a simple affine relation as

$$
\left(\mathbf{h}_{[0 ; 1]}\right)_{k}=0.5 \cdot\left[\left(\mathbf{h}_{[-1 ; 1]}\right)_{k}+1\right] .
$$

It can be easily verified that the two considered criteria, SNR and PBCE, are not modified, since

$$
\left(\hat{\mathrm{h}}_{[0 ; 1]}\right)_{k}=0.5 \cdot\left(\hat{\mathbf{h}}_{[-1 ; 1]}\right)_{k}, \quad \forall k \neq 0,
$$

provided that we impose $\left(\hat{\mathbf{h}}_{[0 ; 1]}\right)_{0}=\left(\hat{\mathbf{h}}_{[-1 ; 1]}\right)_{0}$ (corresponding to a zero-order filtering).

The binary coding performs quite well, especially if we compare its behavior with that of the $4 f$ correlator. ${ }^{10}$ Ternary coding, of course, performs better. But the most dramatic behavior is that of spiral coding. The latter shows excellent performance (it was already interesting in the $4 f$ architecture), close to that of pure-amplitude coding. We better understand this phenomenon when we observe the evolution of the projection angle $\varphi$ (Fig. 10). $\varphi$ evolves according to the filter histogram so that it compensates for the gain variation when the gain parameter 


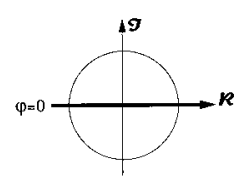

(a)

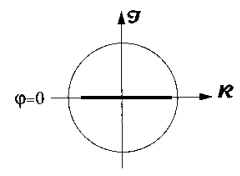

(c)

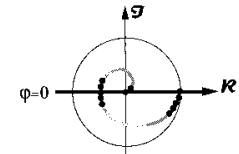

(b)

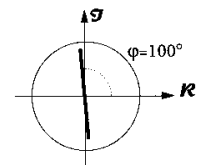

(d)

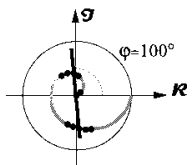

Fig. 10. Complex histograms of OT filters implemented onto a spiral coding domain. The original filter (a) is supported by the real axis (thick line). When one implements a filter onto a spiral, the angle $\varphi$ evolves in the function of the gain parameter $\gamma$ [in (c) $\gamma$ is $40 \%$ smaller than in (a); the histogram is therefore more compact] to make the projection onto the histogram minimize the Euclidean distance between the original filter and its projection onto the spiral. In (b) the implementation is performed with $\varphi=$ 0 ; in (d), the filter has to be dephased by $100^{\circ}$ before projection to make the projection onto the spiral (e) minimize the Euclidean distance.

$\gamma$ varies (without modification of the spectral density shape $\hat{B}_{\mathbf{u}}$ ).

The implementation onto a $360^{\circ}$ spiral is undoubtedly attractive. In future studies it will be interesting to investigate the implementation onto spirals with a different maximum dephasing or onto spirals whose characteristics have been experimentally determined.

\section{Discussion}

In the present paper we have given elements to answer the question, Which coding should be used to best code an OT filter in a JTC? Another important question would be, Which coding should be used to best code the scene in a JTC? Here we discuss the fact that this latter point, which cannot be alleviated, is not within the scope of the present paper. We can, however, notice that such a discussion has been tackled in the case of the $4 f$ correlator. ${ }^{34}$ In the present study the scene is assumed to be noncoded (displayed as is, which is, of course, not possible at the moment with currently available SLM's).

Our purpose was to provide an algorithmic tool for computing efficient implementations of OT filters and to exhibit the interesting potential properties of the typical coding provided by twisted nematic liquidcrystal devices (the spiral coding).

The question of coding the scene, although quite similar to that of coding the filter from the technical point of view, proves to be different. In any case the coding of the scene and of the filter can be considered separately, since two different modulators can be used. The filter and the scene do not usually have the same requirements. The filter exhibits fine details and can be displayed at a low rate [provided a large bank of filters is not used; in that case a multichannel correlator can be considered, or several classical filters can be put together into a composite filter such as a synthetic discriminant function filter ${ }^{7}$. The scene can exhibit many details (but most of them may be noise and so may be undesirable) and must usually be displayed at a high rate (in applications such as tracking, for example). In this case a twisted nematic liquid crystal, whose typical operating rate is below $100 \mathrm{~Hz}^{35}$ (and for most devices even below $25 \mathrm{~Hz}$ ) cannot be used, and the only possible coding domains that can be achieved are the binary or bipolar ones, or a cascade of such devices, ${ }^{36}$ resulting in multiple levels of amplitude and/or phase. In such a case it can prove interesting to dissociate the display of the filter and of the image.

These few elements form only the starting point of an interesting problem: The question of coding the input image deserves further study, which will have to be considered elsewhere.

\section{Conclusion}

The implementation of correlation filters onto coding domains of limited extent is imposed by technology: At the moment no existing spatial light modulator (SLM) is capable of coding any complex value within the unity disk. In the case of the joint transform correlator (JTC) we propose a method that allows a user to code any optimal trade-off (OT) filter (any filter, actually) onto any coding domain in a satisfactory and sometimes optimal way. The method consists of using computer-generated holography (CGH) techniques. Even in the case of restricted coding domains with which classic methods scarcely converge toward a satisfactory solution, our technique, a combination of POCS-like and DBS-like techniques, gives satisfactory results.

This implementation technique also allows us to compare various coding domains: A bank of OT filters is coded onto these domains, and the loci of points described by the values of the criteria for each filter are compared. Analyzing the trade-off between peak sharpness and optical efficiency can seem ambiguous. But the trade-off between noise resistance and optical efficiency, and especially the one between noise resistance and peak sharpness, leads to the conclusion that there is a strict hierarchy, related to the extent of the domain. Binary and ternary codings perform quite well (in fact, far better than in the $4 f$ correlator), partly because of an optimization method dedicated to discrete coding domains. The spiral coding domain, in case of a $360^{\circ}$ phase dynamic range, shows high performance, sometimes close to the one of the filter constrained to $[-1 ; 1]$ and seems quite promising. In any case, as pointed out by de Bougrenet de la Tocnaye and Dupont, ${ }^{15}$ we may expect complex amplitude or pure amplitude SLM's that would remove the need for a genuine coding (only saturation would have to be taken into account). But even in this case it would be interesting to study which SLM's perform better, considering that one SLM is used for the reference and another for the scene and that they do not necessarily have to exhibit the same characteristics. 
Appendix A: GPP, Projection onto a Continuous Domain (Real Only or Phase Only) without Dephasing Optimization

It can be shown with simple considerations that the ideal filter $\mathbf{h}_{\mathbf{u}}{ }^{0}$ is real only and that its optimal implementation onto a real-only coding domain therefore requires no dephasing, unlike its implementation onto a spiral coding domain [such as described in Fig. 1(g) ]. An algorithm for this latter case is developed in Appendix B. It also seems more obvious that the implementation onto a perfect phase-only coding domain [Fig. 1(d)] requires no additional dephasing, because of the circular symmetry of the domain.

1. Let $k=0, \mathbf{h}^{k}=\mathbf{h}_{\mathbf{u}}{ }^{0}, E_{k}=+\infty$. Choose a tolerance coefficient Tol for the error reduction and speed $_{0}$ for the speed of the error reduction. Let speed $=$ speed $_{0}$.

2. Project $\mathbf{h}^{k}$ onto the coding domain:

$$
\mathbf{h}_{p}{ }^{k}=\mathscr{P}\left(\mathbf{h}^{k}\right) .
$$

3. Compute the Fourier transform $\hat{\mathbf{h}}_{p}{ }^{k}$ of $\mathbf{h}_{p}{ }^{k}$.

4. Perform a gradient error reduction:

- Compute error: $E_{k+1}=\Sigma_{n}\left(\hat{B}_{\mathbf{u}}\right)_{n} \mid\left(\hat{\mathbf{h}}_{\mathbf{u}}{ }^{0}\right)_{n}-$ $\left.\left(\hat{\mathbf{h}}_{p}{ }^{k}\right)_{n}\right|^{2}$.

- If $E_{k+1}-E_{k}<$ Tol $E_{k+1}$, go to step 7; else if $E_{k+1}<E_{k}$, perform a gradient reduction:

$$
\begin{aligned}
\hat{\mathbf{h}}^{\mathrm{OK}}= & \hat{\mathbf{h}}_{p}{ }^{2}, \\
\left(\hat{\mathbf{h}}^{k+1}\right)_{n}= & \left(\hat{\mathbf{h}}^{\mathrm{OK}}\right)_{n}+\left[\left(\hat{\mathrm{h}}_{\mathbf{u}}{ }^{0}\right)_{n}\right. \\
& \left.-\left(\hat{\boldsymbol{h}}^{\mathrm{OK}}\right)_{n}\right] \operatorname{speed}\left(\hat{B}_{\mathbf{u}}\right)_{n} ;
\end{aligned}
$$

else let speed $:=$ speed $/ 2$ and perform a gradient reduction:

$$
\begin{aligned}
\left(\hat{\mathbf{h}}^{k+1}\right)_{n}= & \left(\hat{\mathbf{h}}^{\mathrm{OK}}\right)_{n}+\left[\left(\hat{\mathbf{h}}_{\mathbf{u}}{ }^{0}\right)_{n}-\left(\hat{\mathbf{h}}^{\mathrm{OK}}\right)_{n}\right] \\
& \times \operatorname{speed}\left(\hat{B}_{\mathbf{u}}\right)_{n} .
\end{aligned}
$$

5. Compute the inverse Fourier transform $\mathbf{h}^{k+1}$ of $\hat{\mathbf{h}}^{k+1}$

6. Perform $k:=k+1$ and go back to step 2 .

7. $\mathbf{h}=\mathbf{h}_{p}{ }^{k}$.

It must be taken into account that a high speed coefficient can accelerate the whole process; it can also make the convergence more difficult.

\section{Appendix B: GPP, Projection onto a Continuous Domain Such as the Complex Spiral Described in Figure 1}

1. Let $k=0, \mathbf{h}^{k}=\mathbf{h}_{\mathbf{u}}{ }^{0}, E_{k}=+\infty$. Choose a tolerance coefficient Tol for the error reduction and $\operatorname{speed}_{0}$ for the speed of the error reduction. Let speed $=$ speed $_{0}$.

2. $\quad$ Set $\varphi=0, E_{\text {best }}=+\infty$.

3. Project $\mathbf{h}^{k}$ onto the dephased coding domain:

$$
\mathbf{h}_{p}{ }^{k}=\mathscr{P}\left[\mathbf{h}^{k} \exp (i \varphi)\right] .
$$

4. Compute the Fourier transform $\hat{\mathbf{h}}_{p}{ }^{k}$ of $\mathbf{h}_{p}{ }^{k}$.

5. Compute the error caused by projection:

$$
E_{k+1}=\sum_{n}\left(\hat{B}_{\mathbf{u}}\right)_{n} \cdot\left|\left(\hat{\mathbf{h}}_{\mathbf{u}}{ }^{0}\right)_{n}-\left(\hat{\mathbf{h}}_{p}{ }^{k}\right)_{n}\right|^{2} ; \quad \text { if } E_{k+1}<E_{\text {best }},
$$

perform $\varphi_{\text {best }}=\varphi, \quad E_{\text {best }}=E_{k+1}$.

6. Increment $\varphi$ and go back to step 3 , until $\varphi$ $=2 \pi$.

7. Perform a gradient error reduction:

$$
E_{k+1}=E_{\text {best }}, \quad \varphi=\varphi_{\text {best }}, \quad \mathbf{h}_{p}^{k}=\mathscr{P}\left(\mathbf{h}^{k} \exp (i \varphi)\right]
$$

(the filter projection may have been stored instead of being computed again).

If $E_{k+1}-E_{k}<$ Tol, $E_{k+1}$, go to step 10; else if $E_{k+1}$ $<E_{k}$, perform a gradient reduction:

$$
\begin{aligned}
\hat{\mathbf{h}}^{\mathrm{OK}}= & \hat{\mathbf{h}}_{p}{ }^{k}, \\
\left(\hat{\mathbf{h}}^{k+1}\right)_{n}= & \left(\hat{\mathbf{h}}^{\mathrm{OK}}\right)_{n}+\left[\left(\hat{\mathbf{h}}_{\mathbf{u}}{ }^{0}\right)_{n}\right. \\
& \left.-\left(\hat{\mathbf{h}}^{\mathrm{OK}}\right)_{n}\right] \operatorname{speed}\left(\hat{B}_{\mathbf{u}}\right)_{n} ;
\end{aligned}
$$

else let speed $:=$ speed $/ 2$ and perform a gradient reduction:

$$
\left(\hat{\mathbf{h}}^{k+1}\right)_{n}=\left(\hat{\mathbf{h}}^{\mathrm{OK}}\right)_{n}+\left[\left(\hat{\mathbf{h}}_{\mathbf{u}}{ }^{0}\right)_{n}-\left(\hat{\mathbf{h}}^{\mathrm{OK}}\right)_{n}\right] \text { speed }\left(\hat{B}_{\mathbf{u}}\right)_{n} .
$$

8. Compute the inverse Fourier transform $\mathbf{h}^{k+1}$ of $\hat{\mathbf{h}}^{k+1}$.

9. Perform $k:=k+1$ and go back to step 2 .

10. $\mathbf{h}=\mathbf{h}_{p}{ }^{k}$.

\section{Appendix C: GPP + DBS, Projection onto a Discrete} Domain

To simplify the description of the algorithm, we assume that the considered domain is real only. Then no iterative search over $\varphi \in[0,2 \pi]$ is necessary for minimizing the error function. We assume use of $N$ levels of quantization.

1. Compute $N$ levels of quantization:

$$
\begin{aligned}
\{\operatorname{level}[1], \ldots, \operatorname{level}[N]\}=\{ & 0, \frac{1}{N-1}, \ldots, \frac{K}{N-1}, \\
& \ldots, 1-\frac{K}{N-1}, \ldots, 1 \\
& \left.-\frac{1}{N-1}, 1\right\} .
\end{aligned}
$$

Let $k=0, K=1, \mathbf{h}^{k}=\mathbf{h}_{\mathbf{u}}{ }^{0}, E_{k}=+\infty$. Choose a tolerance coefficient Tol for the error reduction and speed $_{0}$ for the speed of the error reduction. Let speed $=$ speed $_{0}$.

2. Project $\mathbf{h}^{k}$ onto the progressive coding domain:

$$
\left(\mathbf{h}_{p}{ }^{k}\right)_{n}= \begin{cases}\left(\mathbf{h}^{k}\right)_{n} & \text { if } \frac{K}{N-1}<\left(\mathbf{h}^{k}\right)_{n}<1-\frac{K}{N-1}, \\ \mathscr{P}\left[\left(\mathbf{h}^{k}\right)_{n}\right] & \text { else }\end{cases}
$$


where $\mathscr{P}$ denotes the Euclidean projection operator onto the progressive domain that consists of $\{\mathbf{l e v}$ $\operatorname{el}[1], \ldots, \operatorname{level}[K], \operatorname{level}[N-K+1], \ldots, \operatorname{level}[N]\}$.

3. Compute the Fourier transform $\hat{\mathbf{h}}_{p}{ }^{k}$ of $\mathbf{h}_{p}{ }^{k}$.

4. Perform a gradient error reduction:

- Compute the error: $E_{k+1}=\Sigma_{n}\left(\hat{B}_{\mathbf{u}}\right)_{n} \mid\left(\hat{\mathbf{h}}_{\mathbf{u}}{ }^{0}\right)_{n}$ $-\left.\left(\hat{h}_{p}^{k}\right)_{n}\right|^{2}$.

- If $E_{k+1}-E_{k}<$ Tol $E_{k+1}$, go to step 7; else if $E_{k+1}<E_{k}$, perform a gradient reduction:

$$
\begin{aligned}
\hat{\mathbf{h}}^{\mathrm{OK}}= & \hat{\mathbf{h}}_{p}{ }^{k}, \\
\left(\hat{\mathbf{h}}^{k+1}\right)_{n}= & \left(\hat{\mathbf{h}}^{\mathrm{OK}}\right)_{n}+\left[\left(\hat{\mathbf{h}}_{\mathbf{u}}{ }^{0}\right)_{n}\right. \\
& \left.-\left(\hat{\mathbf{h}}^{\mathrm{OK}}\right)_{n}\right] \text { speed }\left(\hat{B}_{\mathbf{u}}\right)_{n} ;
\end{aligned}
$$

else let speed $:=$ speed $/ 2$ and perform a gradient reduction:

$$
\begin{aligned}
\left(\hat{\mathbf{h}}^{k+1}\right)_{n}= & \left(\hat{\mathbf{h}}^{\mathrm{OK}}\right)_{n}+\left[\left(\hat{\mathbf{h}}_{\mathbf{u}}{ }^{0}\right)_{n}-\left(\hat{\mathbf{h}}^{\mathrm{OK}}\right)_{n}\right] \\
& \times \operatorname{speed}\left(\hat{B}_{\mathbf{u}}\right)_{n} .
\end{aligned}
$$

5. Compute the inverse Fourier transform $\mathbf{h}^{k+1}$ of $\hat{\mathbf{h}}^{k+1}$

6. Go back to step 2 .

7. If $K=(N-1 / 2)(N$ odd $)$ or if $K=(N / 2)(N$ even), go to step 9 .

8. If $K:=K+1$, perform $\mathbf{h}^{0}=\mathbf{h}^{k}, k=0$ and go to step 2.

9. DBS part: for the sake of clarity let $\mathbf{h}=\mathbf{h}^{k}$ and $E_{\text {prev }}=E_{k+1}, E_{\text {glob }}=E_{k+1}$.

10. Let $i=0$.

11. Let $j=0$, best $=\mathbf{h}_{i}$.

12. If $\left(\mathbf{h}_{i} \neq\right.$ level $\left.[j]\right)$, perform $\mathbf{h}_{i}=$ level $\left.[j]\right)$; else $j:=j+1$. If $j=N+1$, perform $i:=i+1$ and go back to step 11 .

13. Compute the Fourier transform $\hat{\mathbf{h}}$ of $\mathbf{h}$

14. Compute the error $E=\Sigma_{n}\left(\hat{B}_{\mathbf{u}}\right)_{n} \mid\left(\hat{\mathbf{h}}_{\mathbf{u}}{ }^{0}\right)_{n}$ $-\left.(\hat{\mathbf{h}})_{n}\right|^{2}$.

15. If $E<E_{\text {prev }}$, perform best $=\mathbf{h}_{i}, E_{\text {prev }}=E$; else $\mathbf{h}_{i}=$ best.

16. If $j<N$, perform $j:=j+1$ and go to step 12 .

17. If $j=N$ and $i \neq$ length (h), perform $i:=i+1$ and go to step 11 .

18. If $E_{\text {glob }}-E_{\text {prev }}<$ Tol $E_{\text {prev }}$, perform $E_{\text {glob }}$ $=E_{\text {prev }}$ and go back to step 10 .

19. Stop.

The authors thank the anonymous reviewers whose comments helped to make this paper clearer and Richard Juday for valuable suggestions.

\section{References}

1. A. Maréchal and P. Croce, "Un filtre de fréquences spatiales pour l'amélioration du contraste des images optiques," C. R. Acad. Sci. 237, 607-609 (1953).

2. A. VanderLugt, "Signal detection by complex spatial filtering," IEEE Trans. Inf. Theory 10, 139-145 (1964).

3. C. S. Weaver and J. W. Goodman, "A technique for optically convolving two functions," Appl. Opt. 5, 1248-1249 (1966).

4. H. J. Caulfield and W. T. Maloney, "Improved discrimination in optical character recognition,” Appl. Opt. 8, 2354-2356 (1969)
5. Y. N. Hsu and H. H. Arsenault, "Optical pattern recognition using circular harmonic expansion,” Appl. Opt. 21, 4016-4019 (1982).

6. Ph. Réfrégier, "Filter design for optical pattern recognition: multicriteria optimization approach," Opt. Lett. 15, 854-856 (1990).

7. B. V. K. Vijaya Kumar, "Tutorial survey of composite filter designs for optical correlators," Appl. Opt. 31, 4773-4801 (1992).

8. B. V. K. Vijaya Kumar, R. D. Juday, and K. P. Rajan, "Saturated filters," J. Opt. Soc. Am. A 9, 405-412 (1992).

9. R. D. Juday, "Optimal realizable filters and the minimum Euclidean distance principle," Appl. Opt. 32, 5100-5111 (1993).

10. V. Laude and $\mathrm{Ph}$. Réfrégier, "Multicriteria characterization of coding domains with optimal Fourier SLM filters," Appl. Opt. 33, 4465-4471 (1994)

11. B. V. K. Vijaya Kumar, D. W. Carlson, and A. Mahalanobis, "Optimal trade-off synthetic discriminant function filters for arbitrary devices," Opt. Lett. 19, 1556-1558 (1994).

12. J. L. Horner and P. D. Gianino, "Phase-only matched filtering," Appl. Opt. 23, 812-816 (1984).

13. L. P. Yaroslavsky, "Is the phase-only filter and its modifications optimal in terms of the discrimination capability in pattern recognition?" Appl. Opt. 31, 1677-1679 (1992).

14. Ph. Réfrégier, "Optimal trade-off filters for noise robustness, sharpness of the correlation peak, and Horner efficiency," Opt. Lett. 16, 829-831 (1991).

15. J. L. de Bougrenet de la Tocnaye and L. Dupont, "Complex amplitude modulation by use of liquid-crystal spatial light modulators," Appl. Opt. 36, 1730-1741 (1997).

16. U. Mahlab and J. Shamir, "Iterative optimization algorithms for filter generation in optical correlators: a comparison," Appl. Opt. 31, 1117-1125 (1992).

17. M. G. Roe, K. L. Schehrer, R. Dobson, and L. Schirber, "Distortion-invariant optical pattern recognition using composite binary filters," in Optical Pattern Recognition IV, D. P. Casasent, ed., Proc. SPIE 1959, 203-213 (1993).

18. Y. Pétillot, G. Keryer, and J. L. de Bougrenet de la Tocnaye, "Real-time distortion-invariant joint transform correlator using ferroelectric liquid crystal spatial light modulators," in Euro-American Workshop on Optical Pattern Recognition, B. Javidi and $\mathrm{Ph}$. Réfrégier, eds. (SPIE Optical Engineering Press, Bellingham, Wash., 1994), pp. 267-274.

19. B. V. K. Vijaya Kumar, D. W. Carlson, and A. Mahalanobis, "Efficient determination of the optimum gain and angle in the design of optical correlation filters," Opt. Eng. 37, 132-137 (1998).

20. J. J. Burch, "A computer algorithm for the synthesis of spatial frequency filters," Proc. IEEE 55, 599-601 (1967).

21. F. Wyrowski, "Iterative quantization of digital amplitude holograms," Appl. Opt. 28, 3865-3870 (1989).

22. R. W. Gerchberg and W. O. Saxton, "A practical algorithm for the determination of phase from image and diffraction plane pictures," Optik 35, 237-246 (1972).

23. L. M. Bregman, "Finding the common point of convex sets by the method of successive projections," Dokl. Akad. Nauk SSSR 162, 487-490 (1965).

24. H. Stark, W. C. Catino, and J. L. LoCicero, "Design of phase gratings by generalized projections," J. Opt. Soc. Am. A 8, 566-571 (1991).

25. H. Stark and M. I. Sezan, "Image processing using projection methods," in Real-Time Optical Information Processing, B. Javidi and J. L. Horner, eds. (Academic, San Diego, Calif., 1994), pp. 185-232.

26. E. Zhang, S. Noehte, C. H. Dietrich, and R. Männer, "Gradual and random binarization of gray-scale holograms," Appl. Opt. 34, 5987-5995 (1995)

27. M. A. Seldowitz, J. P. Allebach, and D. W. Sweeney, "Synthesis 
of digital holograms by direct binary search," Appl. Opt. 26, 2788-2798 (1987).

28. J. P. Allebach and D. W. Sweeney, "Iterative approaches to computer generated holography," in Computer-Generated Holography II, S. H. Lee, ed., Proc. SPIE 884, 2-9 (1988).

29. B. K. Jennison, J. P. Allebach, and D. W. Sweeney, "Iterative approaches to computer-generated holography," Opt. Eng. 28, 629-637 (1989).

30. L. Legeard, Ph. Réfrégier, and P. Ambs, "Multicriteria optimality for iterative encoding of computer generated holograms," Appl. Opt. 36, 7444-7449 (1997).

31. I. Juvells, A. Carnicer, S. Vallmitjana, and J. Campos, "Implementation of real filters in a joint transform correlator using a positive-only display," J. Opt. 25, 33-40 (1994).

32. M. Taniguchi, K. Matsuoka, and Y. Ichioka, "Computer- generated multiple-object discriminant correlation filters: design by simulated annealing," Appl. Opt. 34, 1379-1385 (1995).

33. W.-H. Lee, "Sampled Fourier transform hologram generated by computer," Appl. Opt. 9, 639-643 (1970).

34. S. Mazé and P. Réfrégier, "Optical correlation: influence of the coding of the input image," Appl. Opt. 33, 6788-6796 (1994).

35. S.-T. Wu, "Nematic liquid crystal," in Spatial Light Modulator Technology: Materials, Devices, and Applications, U. Efron, ed. (Marcel Dekker, New York, 1995), pp. 1-31.

36. S. E. Broomfield, M. A. A. Neil, and E. G. S. Paige, "Programmable multiple-level phase modulation using ferroelectric liquid crystal spatial light modulators," Appl. Opt. 34, 6652-6665 (1995). 